\title{
Gut-Liver Axis, Gut Microbiota, and Its Modulation in the Management of Liver Diseases: A Review of the Literature
}

\author{
Ivana Milosevic ${ }^{1,2, *}$, Ankica Vujovic ${ }^{1,2}$, Aleksandra Barac ${ }^{1,2}$, Marina Djelic ${ }^{3}$, Milos Korac ${ }^{1,2}$, \\ Aleksandra Radovanovic Spurnic 1,2 , Ivana Gmizic ${ }^{2}$, Olja Stevanovic 1,2, Vladimir Djordjevic 1,4, \\ Nebojsa Lekic ${ }^{1,4}$, Edda Russo ${ }^{5}$ (i) and Amedeo Amedei ${ }^{5,6}$ \\ 1 Faculty of Medicine, University of Belgrade, 11000 Belgrade, Serbia; ankica.vujovic88@gmail.com (A.V.); \\ aleksandrabarac85@gmail.com (A.B.); milos.korac@med.bg.ac.rs (M.K.); spurnic@yahoo.com (A.R.S.); \\ stevanovicolja74@gmail.com (O.S.); vladimir.djordjevic@kcs.ac.rs (V.D.); nesalekic67@gmail.com (N.L.) \\ 2 Hospital for Infectious and Tropical Diseases, Clinical Center of Serbia, 11000 Belgrade, Serbia; \\ gmizic_ivana@yahoo.com \\ 3 Faculty of Medicine, Universisty of Belgrade; Institute of Medical Physiology "Rihard Burijan", \\ 11000 Belgrade, Serbia; mdjelic011@gmail.com \\ 4 Clinic for Digestive Surgery, Clinical Center of Serbia, 11000 Belgrade, Serbia \\ 5 Department of Experimental and Clinical Medicine, University of Florence, 50134 Florence, Italy; \\ edda.russo@unifi.it (E.R.); amedeo.amedei@unifi.it (A.A.) \\ 6 Department of Biomedicine, Azienda Ospedaliera Universitaria Careggi (AOUC), 50134 Florence, Italy \\ * Correspondence: ivana.s.milosevic@mfub.bg.ac.rs; Tel.: +381-11-2683-366
}

Received: 22 December 2018; Accepted: 14 January 2019; Published: 17 January 2019

Abstract: The rapid scientific interest in gut microbiota (GM) has coincided with a global increase in the prevalence of infectious and non-infectivous liver diseases. GM, which is also called "the new virtual metabolic organ", makes axis with a number of extraintestinal organs, such as kidneys, brain, cardiovascular, and the bone system. The gut-liver axis has attracted greater attention in recent years. GM communication is bi-directional and involves endocrine and immunological mechanisms. In this way, gut-dysbiosis and composition of "ancient" microbiota could be linked to pathogenesis of numerous chronic liver diseases such as chronic hepatitis $\mathrm{B}(\mathrm{CHB})$, chronic hepatitis $\mathrm{C}(\mathrm{CHC})$, alcoholic liver disease (ALD), non-alcoholic fatty liver disease (NAFLD), non-alcoholic steatohepatitis (NASH), development of liver cirrhosis, and hepatocellular carcinoma (HCC). In this paper, we discuss the current evidence supporting a GM role in the management of different chronic liver diseases and potential new therapeutic GM targets, like fecal transplantation, antibiotics, probiotics, prebiotics, and symbiotics. We conclude that population-level shifts in GM could play a regulatory role in the gut-liver axis and, consequently, etiopathogenesis of chronic liver diseases. This could have a positive impact on future therapeutic strategies.

Keywords: gut microbiota; gut-liver axis; chronic liver diseases; fecal transplantation; probiotics

\section{Gut Microbiota}

The gut microbiota (GM) is a diverse ecosystem that consists of bacteria, protozoa, archaea, fungi, and viruses, which exist in a specific symbiosis between each other and the human body as well. Currently, it is well known that GM plays relevant roles in physiological and pathological conditions of human health, taking part in digestion, vitamin B synthesis, immunomodulation, and promotion of angiogenesis and nerve function. In addition, it is unavoidable that the GM has an impact on pathogenesis of gastrointestinal, hepatic, respiratory, cardiovascular, endocrine, and many other disorders, arising as "a new virtual metabolic organ" [1]. 
The GM colonizes human intestinal tract, which accounts for more than 100 trillion bacteria, and has a complex genome of 150-fold more genes than the human genome [2]. The majority of gut microorganisms cannot be cultured using standard techniques, so the development of culture independent molecular methods based on sequencing of the phylogenetic marker-16S/18S ribosomal RNA offer better insight in the GM structure. The GM is essentially ecomposed of the five phyla-Firmicutes (79.4\%) (Ruminococcus, Clostridium, and Eubacteria), Bacteroidetes (16.9\%) (Porphyromonas, Prevotella), Actinobacteria (2.5\%) (Bifidobacterium), Proteobacteria (1\%), and Verrumicrobia (0.1\%) [3]. Lactobacilli, Streptococci, and Escherichia coli are found in small numbers in the gut. Different genetic and environmental factors influence the GM composition. For example, children born by natural childbirth inherit about $40 \%$ of the mother's intestinal flora, while GM composition is very different after the caesarean section. During the first two years of life, the diet is the most prominent factor that determines GM. Later in life, GM composition depends on age, diet, medications, and the environment.

Studies published in the last decade confirmed that the GM is implicated in the pathogenesis of various diseases, such as cancer and autism, depression, Clostridium difficile infection, inflammatory bowel disease, irritably bowel syndrome, colorectal carcinoma, infectious and non-infectious chronic liver diseases, obesity, diabetes mellitus type 2, atherosclerosis, and chronic kidney diseases [4-9]. In the present review, the important role of GM in the pathogenesis of most common liver diseases was discussed.

\section{Gut-Liver Axis}

GM as a "virtual metabolic organ" makes axis with a number of extraintestinal organs, such as kidneys, brain, cardiovascular, and the bone system, but the gut-liver axis attracts increased attention in recent years [10]. The gut-liver axis is a consequence of a close anatomical and functional, bidirectional interaction of the gastrointestinal tract and liver, primarily through a portal circulation. The symbiotic relationship between the GM and the liver is regulated and stabilized by a complex network of interactions, that encompass metabolic, immune, and neuroendocrine crosstalk between them [4]. The tight junctions (TJ) within the gut epithelium represent a natural barrier to bacteria and their metabolic products [11]. Antigens (Ag) (originating either from pathogenic micro-organisms or from food) that pass through these connections, are recognized by dendritic cells, or activate the adaptive immune system by modulating the $\mathrm{T}$ cell response. Minimal concentrations of pathogen-associated molecular patterns (PAMPs), such as lipopolysaccharides (LPS), peptidoglycans, and flagelin, activate the nuclear factor kappa B (NFK $\beta$ ) through toll-like receptors (TLRs) and nod-like receptors (NLRs), which leads to the production of inflammatory cytokines and chemokines that enter portal circulation. In addition to hepatocyte damage, PAMPs can activate stellate cells involved in fibrosis promotion and progression, while Kupffer cells are even more responsive to LPS than hepatocytes [12]. Since the gut-liver axis affects the pathogenesis of liver diseases, it is an important focus of current clinical research (Scheme 1). 


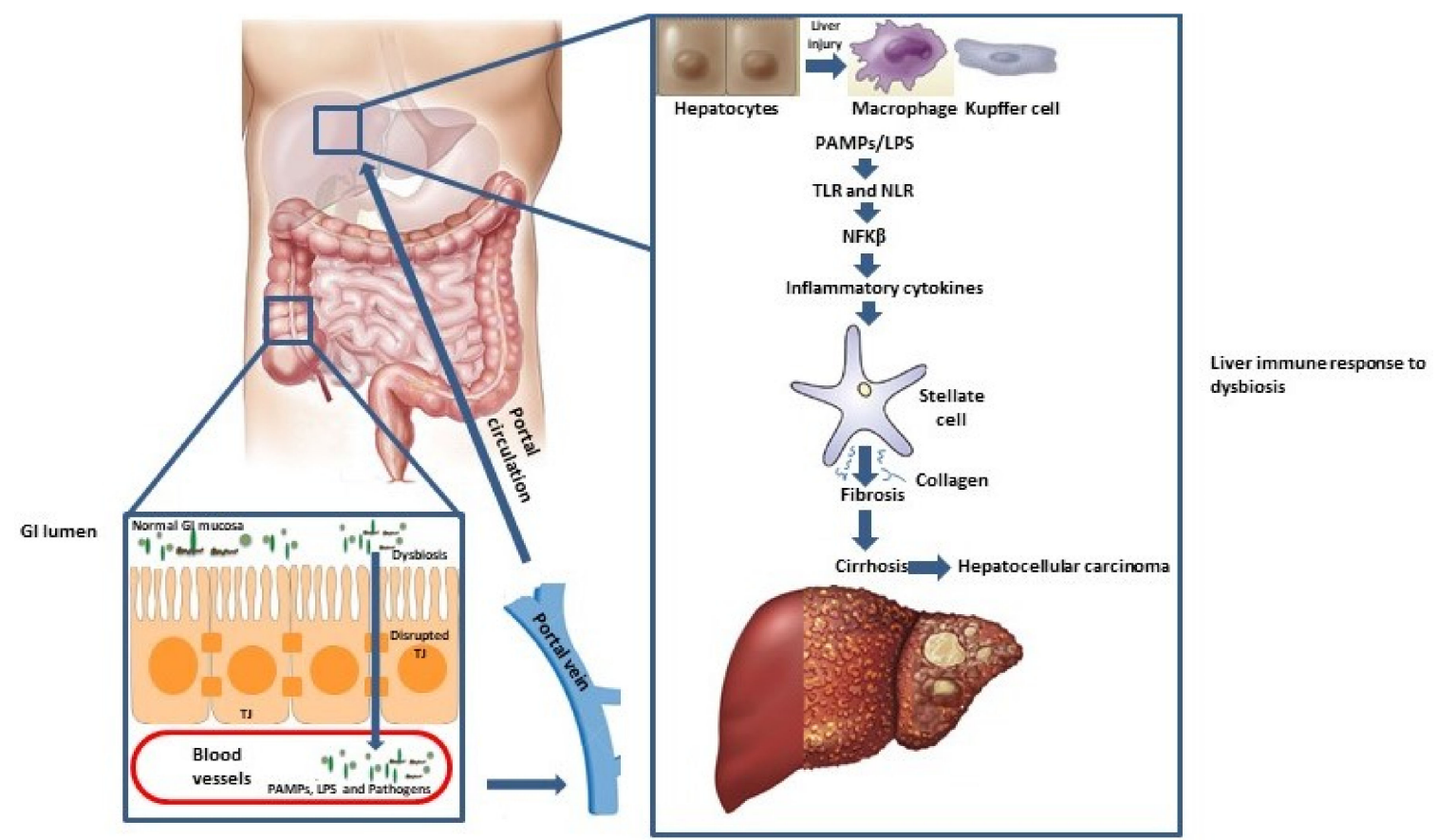

Scheme 1. Gut-liver axis pathogenesis. Abbreviations: GI, gastrointestinal, LPS, Lipopolysaccharides, NFK $\beta$, nuclear factor kappa B, NLR, Nod-like receptors, PAMPs, Pathogen-associated molecular patterns, TJ, Tight junctions, TLR, Toll-like receptors.

\section{Disbyosis and Liver Diseases}

The gut-liver axis has an impact on pathogenesis of numerous chronic liver diseases such as chronic hepatitis $\mathrm{B}(\mathrm{CHB})$, chronic hepatitis $\mathrm{C}(\mathrm{CHC})$, alcoholic liver disease (ALD), non-alcoholic fatty liver disease (NAFLD), non-alcoholic steatohepatitis (NASH), development of liver cirrhosis, and hepatocellular carcinoma (HCC) (Table 1).

Table 1. Gut microbiota-associated liver diseases.

\begin{tabular}{|c|c|c|}
\hline Disease & Dysbiotic Features & References \\
\hline СНB & $\begin{array}{c}\text { Decreased ratio of Bifidobacteriacae /Enterobacteriaceae: } \\
\text {-low levels of Bifidobacteria and Lactobacillus } \\
\text {-high levels of Enterococcus and Enterobacteriaceae }\end{array}$ & [13] \\
\hline HBV related cirrhosis & $\begin{array}{l}\text { Decreased Bacteroidetes } \\
\text { Increased Proteobacteria }\end{array}$ & [14] \\
\hline $\mathrm{CHC}$ & $\begin{array}{c}\text { Decreased Bifidobacterium } \\
\text { Increased Prevotella and Faecalibacterium }\end{array}$ & [15] \\
\hline HCC & $\begin{array}{c}\text { Decreased Lactobacillus spp., Bifidobacterium spp. and Enterococcus spp. } \\
\text { Increased Escherichia coli }\end{array}$ & $\begin{array}{l}{[16]} \\
{[17]}\end{array}$ \\
\hline HE & $\begin{array}{l}\text { Production of ammonia and endotoxins by urease-producing bacteria, } \\
\text { such as Klebsiella and Proteus }\end{array}$ & [18] \\
\hline ALD & $\begin{array}{l}\text { Decreased levels of butyrate-producing Clostridiales species } \\
\text { Increased levels of pro-inflammatory Enterobacteriaceae }\end{array}$ & [19] \\
\hline NAFLD/NASH & Increased Firmicutes / Bacteroidetes ratio & {$[20,21]$} \\
\hline Cirrhosis & $\begin{array}{l}\text { Decreased Bacteroidetes and Firmicutes } \\
\text { Increased Streptococcus spp. and Veillonella spp. }\end{array}$ & $\begin{array}{l}{[22]} \\
{[16]}\end{array}$ \\
\hline
\end{tabular}

Abbreviations: ALD, alcoholic liver disease, $\mathrm{CHB}$, chronic hepatitis B, CHC, chronic hepatitis C, HBV, hepatitis B virus, HCC, hepatocellular carcinoma, HE, hepatic encephalopathy, NAFLD, non-alcoholic fatty liver disease, and $\mathrm{NASH}$, non-alcoholic steatohepatitis.

In general, an increased intestinal permeability and bacterial translocation could enable microbial metabolites to reach the liver, which would impair the bile acid (BA) metabolism and promote gut dysmotility and systemic inflammation. All these conditions could induce gut dysbiosis, which, in turn, 
further increases liver damage. It has been observed that the stage of liver injury correlates closely with the severity of gut dysbiosis [23]. Alterations in the fecal bacterial flora are described by changes in the composition of the dominant Bacteroidetes and Firmicutes phyla, including Ruminococcaceae, Lachnospiraceae, and Clostridiales, which produce short-chain fatty acids (SCFA) that are an energy source for the intestinal epithelium's cells, but can also regulate secondary BA metabolism and induce a regulatory immune process and IgA production.

\subsection{Hepatitis B Virus (HBV) Infection}

$\mathrm{CHB}$ is an important health issue worldwide. Acute HBV infection leads to $\mathrm{CHB}$ in just $5 \%$ of adult patients, while the proportion is quite different in children, since more than $90 \%$ of exposed neonates and $30 \%$ to $50 \%$ of children aged 1 to 5 years fail in HBV clearance. Liver injury is mediated by HBV induced immune response. TLRs play an important role in the production of interferons and proinflammatory cytokines and immune cells recruitment in order to suppress viral replication. It has been established that age-specific seroclearance depends not only on the maturity of the immune system, but on the GM stability as well [24]. Involvement of GM in HBV clearance was demonstrated in animal models. Chou et al. showed that adult mice with mature GM managed to clear HBV after six weeks of infection, which is the opposite among young mice without GM, who remained HBV positive. The fact that adult mice failed to clear HBV after gut sterilization by antibiotics (6 to 12 weeks), emphasizes the GM significance in anti-HBV immunity [25]. It also implies new therapeutic strategy for patients with HBV infection [26]. In fact, the transplantation of fecal microbiota (FMT), in addition to standard antivirals, has been shown to be effective in HBeAg clearance [27].

Compositional and structural changes of GM have been detected in patients with $\mathrm{CHB}$ and liver cirrhosis. These patients have a decreased ratio of Bifidobacteriaceae/Enterobacteriaceae (B/E), based on low levels of Bifidobacteria and Lactobacillus, and high levels of Enterococcus and Enterobacteriacea. In addition, gut permeability is altered when accompanied with bacterial translocation and the presence of endotoxins in the portal vein, which leads to increased TLS/NLR activation in the liver with consequential cytokine production and occurrence of liver lesions, progression of fibrosis, and development of cirrhosis and HCC [2,13]. Wei et al. have demonstrated that GM of patients with HBV-related cirrhosis contained lower levels of Bacteroidetes (4\% vs. 53\%) and increased levels of Proteobacteria (43\% vs. $4 \%$ ) compared to the heathy group [14]. In an other study, patients with alcohol-related and HBV-related cirrhosis showed decreased GM diversity, compared to healthy individuals, with a predominance of Enterobacteriaceae and Streptococcaceae [28].

\subsection{Hepatitis $C$ Virus (HCV) Infection}

$\mathrm{CHC}$ is a global health problem that leads to progressive liver fibrosis and the cirrhosis development in $20 \%$ to $30 \%$ of untreated patients after 20 to 30 years. It has been estimated that $1 \%$ to $4 \%$ of these patients develop HCC each year [29]. GM has been rarely analyzed in patients with $\mathrm{HCV}$ infection. According to published data, the GM found in HCV patients shows lower microbial diversity in comparison to those in healthy controls $[15,28,30]$. $\mathrm{CHC}$ could alter microbiota composition through IgA produced by HCV infected gastric B-lymphocyte. GM found in Egyptian patients with CHC contains more Prevotella and Faecalibacterium and less Acinetobacter, Veillonella, and Phascolarctobacterium than healthy individuals. In the study of Aly et al., Bifidobacterium was detected only in GM of the healthy group, posing the possible new role of Prevotella/Faecalibacterium vs. Bifidobacterium ratio as a biomarker for $\mathrm{CHC}$ and fibrosis progression [15]. Disease progression could bring more profound changes in CHC patients' GM. Therefore, according to Heidrich et al., decreased diversity was more pronounced in $\mathrm{HCV}$ patients' with established cirrhosis than in those with less advanced CHC [31]. Liver cirrhosis per se could be an independent risk factor for dysbiosis regardless of the HCV viral load. This hypothesis is in agreement with a study performed by Bajaj et al. who found that patients with HCV cirrhosis have gut dysbiosis regardless of long-term HCV eradication. A sustained virological response (SVR) did not improve gut dysbiosis in patients with 
HCV cirrhosis, due to refractory systemic inflammation and endotoxemia in these individuals [32]. Bacterial translocation was described in patients with $\mathrm{CHC}$ and together with increased intestinal permeability ("leaky gut"), it poses a well-established milieu with TLR/NLR activation and expression of pro-inflammatory cytokine genes, especially in those with cirrhosis [33].

\subsection{Alcoholic Liver Disease}

Alcohol abuse is a prominent cause of liver damage worldwide. GM is recognized as a key player in the severity of liver injury in ALD, in addition to the quantity of consumed alcohol and genetic predisposition (patatin like phospholipase domain containing 3 (PNPLA3), Transmembrane 6 superfamily 2 human gene (TM6SF2), membrane bound O-acyltransferase domain containing 7 (MBOAT7), and solute carrier family 38 member 4 (SLC38A4) etc.) [34]. Alcohol consupmtion leads to small and large intestine overgrowth and modulation of GM composition in both animals and humans [35]. Alcohol and its degradation products disrupt epithelial TJ leading to increased intestinal permeability and inflammation [34]. Gut-derived PAMPs (e.g., endotoxin) are increased after heavy alcohol intake [36]. Ethanol consumption alters the GM composition through SCFAs modulation. Intestinal levels of SCFAs are lower after alcohol consumption with the exception of increases in acetic acid levels, which is the metabolite of ethanol [37]. Alcoholic abuse was shown to be associated with decreased levels of butyrate-producing Clostridiales species order and increased levels of pro-inflammatory Enterobacteriaceae. In those with established cirrhosis, multiple members of the Bacteroidales order were depleted with a rise of taxa normally inhabiting the oral cavity [19].

\subsection{Non-Alcoholic Fatty Liver Disease and Non-Alcoholic Steatohepatitis}

NAFLD is one of the most important causes of liver disease worldwide, with global prevalence of $25 \%$. NAFLD is one of the top risk factors for HCC and is predicted to become the most common indication for liver transplantation [38]. NAFLD is a consequence of triglyceride accumulation in the hepatocytes and is considered to be the hepatic manifestation of obesity and the metabolic syndrome [39]. About $20 \%$ of patients with NAFLD develop NASH, which is a chronic hepatic inflammation that can progress to cirrhosis, end stage liver disease (ESLD), and HCC. Pathogenesis of NASH is not yet fully elucidated, but it is described as a "two hit" phenomenon. The primary event is lipid accumulation with alterations of lipid homeostasis associated with obesity, insulin resistance, and adipokine abnormalities. The second "hit" is a combination of oxidative stress, lipid peroxidation, mitochondrial dysfunction, BA toxicity, cytokine-mediated recruitment, and retention of inflammatory cells [40]. Obesity is associated with dysbiotic gut microbiota, with decreased diversity and an increased Firmicutes / Bacteroidetes ratio [20]. A similar Firmicutes / Bacteroidetes ratio was found in diabetes patients as well. Endogenous ethanol is constantly produced by microbiota, regardless of oral alcohol intake, especially in those with a sugar-rich diet. Increased ethanol production by microbiota in obese humans and mice leads to the activation of TLRs in the liver, cytokine production, and alters the BA profile. Endogenous ethanol serum concentrations are significantly higher in patients with NASH compared to obese or healthy controls [41]. Gut dysbiosis in patients with NAFLD/NASH promotes insulin resistance, de novo lipogenesis in liver, and also increases intestinal permeability, which promotes chronic PAMPs exposure and oxidative stress caused by increased endogenous ethanol [21]. Endotoxin/TLR4 signalling contributes to the development of fibrosis and progression to cirrhosis through hepatic stellate cell activation. The GM plays a critical role in the conservation of the mainstream BA pool, which transforms BA to several metabolites by oxidation/epimerization, deconjugation, esterification, 7-dehydroxylation, and desulfatation. Changes in any of these modulations is a cause of disease. GM dysbiosis in NAFLD could affect the conversion of primary bile acids into secondary bile acids [42]. It has been observed that the bacteria able to make this transformation are decreased in the NAFLD cirrhosis fecal samples. In particular, there is a higher level of Enterobacteriaceae (that could be potentially pathogenic) with lower Ruminococcaceae, Lachnospiraceae, and Blautia (with a $7 \alpha$-dehydroxylating activity) abundances. 
Currently, the roles of BA and nuclear receptors are also strongly considered. The BA primarly synthesized in the liver are secreted to the gallbladder and then released into the duodenum following food ingestion. In the gut, the size and composition of the BA pool can be modified by GM via the biotransformation of primary into secondary BA. The BA contributes to the emulsification and fat solubilization but also activates the expression of a nuclear bile acid receptor FXR (farnesoid X receptor) and a membrane $G$ protein-coupled receptor TGR5. The reduction of the secondary BA synthesis attributed to GM dysbiosis lowers the activation of nuclear receptors FXR and TGR5 in the ileum, which leads to retained bile salts, perpetuation of gut permeability, small bowel translocation, and bacterial overgrowth, which contributes to liver disease [43]. The FXR plays a key role in mediating the crosstalk between the host and GM, especially through the modulation of enterohepatic BA circulation. FXR exerts bile-acid regulatory effects via a tissue-specific mechanism. [44]. In detail, in the liver, FXR induces the expression of the small heterodimer partner (SHP), which inhibits CYP7A1 (Cholesterol 7 alpha-hydroxylase) expression, while, in the intestine, FXR increases the levels of circulating fibroblast growth factor 19 (FGF19), which decrease the expression of CYP7A1 and cytochrome P450 12a-hydroxylase B1 (CYP8B1). Therefore, this leads to the inhibition of BA synthesis [45].

FXR activation has been known to reduce triglyceride levels suppressing the synthesis and uptake of the fatty acids in the liver [46]. In addition, the FXR roles in decreasing inflammation have been emerging [47]. Lastly, related to glucide metabolism, FXR reduces insulin resistance, gluconeogenesis, increases glycogenesis, and, therefore, decreases the blood glucose amount. A more fine elucidation of FXR functions in liver and intestine needs further research. Furthermore, mice models showed that FXR activation, induced by BA products (converted by GM) might protect against bacterial overgrowth, gut permeability, and small bowel translocation [48]. The degree of FXR activation could be regulated by GM dysbiosis, inducing BA alteration and, hence, liver disease secondary to retained bile salts and a leaky gut. Bacteria, translocated from the gut, may additionally decrease FXR activation within hepatocytes, which leads to decreased BSEP (bile salt export pum) activity.

The bile acid-activated FXR and TGR5 has a protective role in liver disease progression, which means the activation of both receptors has been proposed as therapy. Many activators of FXR and TGR5 have been developed from bile acid analogues, which are able to decrease hepatic steatosis and inflammation [49]. FXR agonists such as obeticholic acid have already been recognized as a new therapeutic venue for NASH and cholestatic diseases. NASH patients exhibit increased fecal primary BAs, primary/secondary BAs fecal ratio, and plasma and hepatic BAs concentrations [50]. This cytotoxicity can lead to NASH progression and finally to liver cirrhosis.

\subsection{Hepatic Encephalopathy (HE) and Spontaneous Bacterial Peritonitis (SBP)}

In all patients with ESLD, regardless of its etiology, HE and SBP commonly occur. HE is considered to be a consequence of high ammonia level, but GM and bacteria's products, such as amino acid metabolites (indoles, oxindoles) and endotoxins, are also involved in HE development. The connection between HE and GM is the production of ammonia and endotoxins by urease-producing bacteria, such as Klebsiella and Proteus, which are present in GM [18]. It has been shown that microbiota of the sigmoid colon in liver cirrhosis patients differs in those with HE when compared with patients without HE [51]. Gut dysbiosis in liver cirrhosis also contributes to the development of Spontaneous Bacterial Peritonitis (SBP) through damaged intestinal barrier and a higher degree of microbial translocation [52].

\subsection{Hepatocellular Carcinoma}

HCC is the most common primary malignancy in adults with chronic liver disease and liver cirrhosis [53]. HBV and HCV infection, alcohol abuse, and dietary aflatoxin are major risk factors for the development of HCC. Although HBV and HCV account for $80 \%$ to $90 \%$ of overall HCC, regarding the obesity pandemic, an emergance of potent direct acting antivirals for $\mathrm{HCV}$ and worldwide available vaccine for HBV, it can be expected that HCC epidemiology will change in the future [54]. An alarming 
rise in the incidence of NAFLD and NASH was accompanied by an increased development of NASH-related HCC incidence [55].

All previously mentioned mechanisms, leaky gut, endotoxemia, TLR, dysbiosis, and immunomodulation promote the development of HCC [56]. The gastrointestinal tract contributes to homeostasis by maintaining an intact barrier against LPS and intestinal bacteria. In the case of increased intestinal permeability, bacterial translocation and LPS accumulation will lead to intestinal bacterial overgrowth and changes of GM composition. In patients with chronic liver diseases/cirrhosis, detoxification, degradation, and clearance of LPS and other bacterial products is compromised [57]. Altered microbiota is generally presented in HCC patients [58]. HCC patients have been reported to have high levels of Escherichia coli and other gram-negative bacteria in GM, which are associated to increased LPS serum levels [16]. Oribacterium and Fusobacterium are the most commonly isolated bacteria from tongue swab of HCC patients. On the other side, GM of HCC patients contains reduced levels of Lactobacillus spp., Bifidobacterium spp., and Enterococcus spp. [17]. Unlike merely microbial species, it was shown that microbial metabolism, iron transport, and energy-producing system significantly differ between GM of HCC patients and healthy control [58]. TLR4 expressed by activated stellate cells react to low LPS concentrations, which ensures the development of fibrosis and cirrhosis. The significance of GM and TLR4 activation in hepatocarcinogenesis have been studied in an animal model. It has been demonstrated that GM and TLR4 activation promote HCC development by increased cell proliferation and suppression of apoptosis [59].

\section{Current Perspectives on the Therapeutic Options}

Dietary modification is the focus for many studies on GM modification, but results are not encouraging due to poor compliance [11]. Different treatments options have been developed in the last decade in the attempt to modify pathogenesis factors involved in GM-liver axis, but results are still unsatisfactory [60]. Antibiotics, probiotics, prebiotics, and symbiotics are gaining increasing importance as a treatment option for GM manipulation and its impact on different liver diseases (Table 2).

Table 2. Therapeutic options for gut-microbiota alteration in liver diseases.

\begin{tabular}{|c|c|c|}
\hline Disease & Therapeutic Option & References \\
\hline NAFLD/NASH & $\begin{array}{l}\text {-"VSL \#3“ (Streptococcus thermophilus, Bifidobacterium breve, B. longum, B. infantis, Lactobacillus } \\
\text { acidophilus, L. plantarum, L. paracasei, L. bulgaricus) reduces liver injury }\end{array}$ & {$[61,62]$} \\
\hline Cirrhosis & $\begin{array}{c}\text {-Diet rich in fermented milk, vegetables, cereals, coffee, and tea is associated with a higher } \\
\text { microbial diversity and lower risk for cirrhosis progression }\end{array}$ & [63] \\
\hline \multirow[t]{2}{*}{ HCC } & $\begin{array}{l}\text {-Probiotics can contribute to the inhibition of aflatoxin B-induced hepatocarcinogenesis, } \\
\text { restore intestinal dysbiosis, reduce LPS levels and decrease tumor size }\end{array}$ & \\
\hline & -Probiotic fermented milk and chlorophyllin slow down tumor growth and volume for $40 \%$ & [64] \\
\hline HE & $\begin{array}{l}\text {-Lactobacillus, Bifidobacterium, non-pathogenetic strains of Escherichia coli, Clostridium butyticum, } \\
\text { Streptococcus salivarius, Saccharomyces boulardii and VSL\#3 improve HE }\end{array}$ & [65-69] \\
\hline
\end{tabular}

Abbreviations: NAFLD, non-alcoholic fatty liver disease, HCC, hepatocellular carcinoma, HE, hepatic encephalopathy, and NASH, non-alcoholic steatohepatitis.

\subsection{Antibiotics, Probiotics, Prebiotics, and Symbiotics}

Positive effects of antibiotics on small-intestinal-bacterial-overgrowth related liver damage was shown in the recent literature, which confirms the relationship between GM and liver diseases [70]. Probiotics are micro-organisms with a healthy impact to the human, while pre-biotics are food ingredients, which can not be digested and, on that way, help gut peristaltic and selectively stimulate growth of intestinal bacteria. Some prebiotic food products, like pectin, have been shown to prevent liver injury in rodent models by restoring the levels of Bacteroides and appears to be a promising therapeutic agent [71]. New term "symbiotics" represent combinations of a probiotic and prebiotic. From this group, the most potential for being used as potential treatment of chronic liver diseases have probiotics [11,70]. Probiotics have a significant influence on the gut-liver axis, including the 
immunomodulatory and anti-inflammatory role on intestinal microflora and intestinal barrier function, but also metabolic effects on non-gastrointestinal organs and systems.

Not all probiotic species have the same impact on GM modulation. For example, different strains of Lactobacillus spp. are associated with divergent obesity consequences, pro-obesity and anti-obesity [72]. A study on murine diet-induced obesity showed the opposite metabolic outcomes after different targeted GM manipulation with vancomycin vs. probiotic strain Lactobacillus salivarius, but microbiota changes were similar. On the other side, a recent human study investigating the effect of the Lactobacillus salivarius Ls-33 on a series of inflammatory biomarkers in obese adolescents could not detect any significant impact on the metabolic syndrome [73,74]. These results suggest that no generalization could be done. Use of probiotics and antimicrobials in chronic liver diseases should be determined in accordance to specific gut-liver axis pathology. Although there are a number of findings from animal and human studies, this topic requires more case-control prospective studies with a significant number of patients.

\subsection{Fecal Microbiota Transfer (FMT)}

Next to probiotics and prebiotics, fecal microbiota transfer attracts a lot of attention currently as a possible option for GM editing. FMT is the introduction of a fecal suspension derived from a healthy donor into the intestinal tract of the patient. Animal studies revealed altered GM composition after FMT in alcohol-sensitive mice and prevented alcohol-induced liver lesions [71]. Wang et al. showed potential protective effects of FMT in the improvement of motor activity in a rat model of HE. In addition, FMT had a superior impact on alteration of the intestinal mucosal barrier function, in comparison to probiotics [75]. A different study with the rat model of HE found that rectal administration of a Lactobacillus species suppressed bacterial cell proliferation [76]. Regarding the clinical trial, a pilot study was performed in eight male patients with severe alcoholic hepatitis (SAH) and compared outcomes with historical controls. Researchers found that one week of FMT was effective and safe in SAH patients and improved the values of liver disease severity and survival at one year [77]. In addition, an open label randomized clinical trial was performed in 20 male patients with cirrhosis and recurrent HE despite treatment with lactulose and rifaximin, comparing FMT vs. standard of care [78]. The result showed that FMT from a rationally selected donor (chosen for having the highest abundance of Lachospiraceae and Ruminococcaceae in a universal stool bank) improved cognition, reduced hospitalizations, and ameliorated the dysbiosis condition in cirrhosis with recurrent HE. However, it is not yet known what consequences FMT may excert on dysbiosis in the upper gastrointestinal tract, which may be more involved in liver disease pathogenesis. Further clinical trials benefiting FMT for management of chronic liver diseases are ongoing. It is important to highlight that FMT is a promising therapy to restore a healthy microbiota. However, its safety profile among vulnerable liver disease patients that are usually immunosuppressed and, so at risk of bacteremia, due to bacterial translocation, is unknown.

\subsection{NAFLD}

Several pharmacologic treatments for NAFLD exist, but a standard treatment is not yet established [79,80]. Recent animal model-studies on NAFLD and HE reported beneficial therapeutic effects of probiotics [81-83]. In mice models, probiotics had positive effects on oxidative and inflammatory liver damage mediated by c-Jun N-terminal kinase (JNK) and NF-KB correlated with TNF- $\alpha$ regulation and insulin resistance. The results showed improvement of histological findings including reduced fat deposits and liver damage, with decreased serum alanine aminotransferase (ALT) levels $[81,83,84]$. Apart from animal studies, several clinical trials of probiotics administered to patients with NAFLD have been reported. The mixture of Streptococcus thermophilus, Bifidobacterium breve, Bifidobacterium longum, Bifidobacterium infantis, Lactobacillus acidophilus, Lactobacillus plantarum, Lactobacillus paracasei, and Lactobacillus bulgaricus has joint name "VSL \#3" and represent the most-studied probiotic [61]. A meta analysis 
of effects of probiotic on NAFLD/NASH showed that probiotic therapies can reduce liver aminotransferases, total-cholesterol, TNF- $\alpha$, and improve insulin resistance in NAFLD/NASH patients [62]. Administration of non-complete VSL \#3 (only Lactobacillus bulgaricus and Streptococcus thermophilus) decreased ALT and aspartate aminotransferase (AST) levels, but had no impact on cardiometabolic risk factors [85]. Ma et al. found that the probiotic therapy significantly decreased the levels of ALT, AST, total-cholesterol, high-density lipoprotein (HDL), and TNF- $\alpha$ in the serum, and the homeostasis model assessment of insulin resistance [62]. Comparing single strains, Bifidobacterium longum showed more positive effects than Lactobacillus acidophilus in NAFLD treatment and these beneficial effects correlated to GM modifications [61]. It was demonstrated that GM modulation by linoleic acid and Bifidobacterium resulted in an increase of conjugated linoleic acid (CLA) and altered fatty acid composition of liver [86]. Consequently, by producing CLA as a microbial metabolite, VSL \#3 correlates with NAFLD's improvement [86]. In additional studies (of Malaguarnera et al. and Tanock et al.), probiotics were used with prebiotics in NAFLD patients, and the combination significantly reduced the AST and ALT levels and liver steatosis [87,88].

Prebiotics also hold promise for the NAFLD management. In animal models, the administration of prebiotics could lead to reduced liver inflammation in obese mice through a glucagon-like peptide-2-dependent effect on the gut barrier [89]. There is convincing evidence from short-term high quality human trials supporting the use of dietary prebiotics as a potential therapeutic intervention for NAFLD. However, further studies are needed to correlate these findings with changes in GM [90]. Although most of the conducted studies have limitations including small sample sizes and a lack of data about the patients' diets and life style, the treatments with probiotics and prebiotics for patients with NAFLD are promising [91].

Moreover, a recent meta-analysis study demonstrated that, in addition to probiotics and prebiotics, $\omega-3$ supplementation could have effects on the gut microbiota, which improves NAFLD [92]. It has been shown that n-3polyunsaturated fatty acids (n-3 PUFAs) decrease Akkermansia, Epsilon proteobacteria, Bacteroides, and increase Clostridia [93]. Lastly, the n-3 PUFAs administration has been related to an increment of butyrate-producing bacteria [94].

\subsection{Cirrhosis}

Qin at el. showed reduced Bacteroidetes and Firmicutes levels and increased Streptococcus spp. and Veillonella spp. levels in GM by metagenomics analyses in cirrhotic patients, compared to a healthy population [22]. Streptococcus spp. and Veillonella spp. are bacteria regularly living in oral cavity, which implicates that an increased number of oral bacteria in GM may be related to cirrhosis development [16]. It was reported that the gut microbiome plays a role in the CHB progression to severe liver failure, including inflammation and pathogenic metabolic accumulation [75]. The connection between GM and cirrhosis was recently confirmed in the study of Bajaj et al., which showed a significant improvement of diversity and symbiosis of GM after liver transplantation in a patient with severe cirrhosis [95]. In the recent international cirrhosis cohort study, Bajaj et al. showed that diverse GM achieved by diet is associated with a lower risk for cirrhosis progression and reduced risk of hospitalization [63]. Although the functions of these bacteria in the pathogenesis and complications of liver cirrhosis is not yet clear, these findings give hope for a new therapeutic strategy against liver cirrhosis by focusing on the GM modulation.

\subsection{Hepatic Encephalopathy}

Probiotics inhibit the activity of bacterial ureases, modulate intestinal $\mathrm{pH}$ values, and reduce ammonia absorption in HE [96]. Administration of probiotics and prebiotics could improve HE by altering GM [18]. It is already shown that Lactobacillus, Bifidobacterium, non-pathogenic strains of Escherichia coli, Clostridium butyricum, Streptococcus salivarius, and Saccharomyces boulardii, and VSL \#3 altered GM composition and improve HE [97]. A number of conducted clinical trials indicate that probiotics could be helpful in overt HE [65-69]. In a systematic review of McGee et al., it was 
found that patients treated with probiotics appeared to have reduced plasma ammonia concentrations compared to patients treated with placebo or no intervention. On the other side, treatment without probiotics and synbiotics did not significantly alter mortality and quality of life [65]. The possible explanation is that the sample of study groups was small in the reviewed control trials, as well as quantities of the probiotics were not uniform. Use of probiotics for the secondary HE prophylaxis was studied by Agrawal et al. Although not statistically significant, the occurrence rate of recurrent HE was lower in patients who received probiotics or lactulose compared to those without treatment ( $34 \%$ and $27 \%$, respectively, vs. $57 \%$ ) [67]. In another prospective study by Lunia et al., it was shown that probiotics could be effective in preventing overt HE. Patients with liver cirrhosis who had not experienced overt HE and who received the probiotics were less likely to develop overt HE compared to controls (1.2\% vs. 19\%) [67]. Among the various probiotics, the most efficacious species for HE appeared to be Lactobacilli and Bifidobacteria [97]. The most studied probiotic is the Lactobacillus GG AT strain 53103 (LGG). Safety and tolerability of administrated therapy is especially important for the patients with cirrhosis and HE. It is shown that LGG is safe and well tolerated in patients with cirrhosis and could cause reductions of the endotoxemia and TNF- $\alpha$ production [98]. However, due to a specific clinical course, randomized controlled trials are needed before probiotics can be routinely used in patients with HE.

\subsection{HCC}

The mouse model has shown that the obesity-induced intestinal microbial dysbiosis can lead to HCC [99]. Reports of the therapeutic prevention of HCC using probiotics are limited to aflatoxin-induced HCC. Findings of a clinical and animal model studies suggested that probiotics can contribute to the inhibition of aflatoxin B-induced hepatocarcinogenesis, restore intestinal dysbiosis, reduce LPS levels, and decrease the tumor size [59,100]. An animal study regarding the potential of Lactobacillus plantarum, isolated from Chinese traditional fermented foods, in reducing the toxicity of aflatoxin B1, showed that L. plantarum C88 treatment increased fecal aflatoxin B1 excretion and regulated defense system's deficit of antioxidant in the mice model [100]. Lastly, a study with an HCC rat model showed that probiotic fermented milk and chlorophyllin slow down tumor growth and volume for $40 \%$, by reducing expressions of c-myc, bcl-2, cyclin D1, and rasp- 21 . After treatment with probiotics, mice had high levels of Prevotella and Oscillibacter in their fecal microbiota [64]. Dapito et al. also found that gut sterilization and TLR4 inactivation reduce HCC by $80 \%$ to $90 \%$ and could serve as potential HCC prevention strategies [59].

\section{Conclusions}

Knowledge regarding the gut-liver axis has improved in the last decade. It is confirmed that gut microbiota has a strong relationship with liver and plays a significant role in the pathogenesis of chronic liver diseases, including NAFLD, fibrosis progression, CHB, cirrhosis, HE, HCC, etc. However, the mechanism of this connection in different liver diseases is still unclear. Our knowledge about the clinical significance of probiotics' use in liver disease is starting to take shape. Although clinical and experimental studies confirmed the therapeutic potential of probiotics in chronic liver diseases, data on safety assessments, and impact on microbiota-host interactions are missing. Recently, many animal studies tried to reveal these gaps, but differences in physiology and variations in the molecular targets between mice and humans can lead to translational limitations. Large prospective controlled studies with a standardized dose of probiotics therapy and duration, liver biopsy, and patients' follow-up appointments are required to confirm these encouraging results.

Author Contributions: Conceptualization: I.M., A.V., and A.B. Writing—original draft preparation: M.Dj., M.K., A.R.S., I.G., O.S., and E.R. Writing-review and final editing: I.M., A.V., A.B., and A.A. Funding acquisition and critical reading: V.Dj. and N.L., A.A. initiated and supervised the whole work. 
Funding: Marina Djelic and Ankica Vujovic received support for research from the Project of Ministry of Education, Science and Technology of the Republic of Serbia (No. III41025). Aleksandra Barac received support for research from the Project of Ministry of Education, Science and Technology of the Republic of Serbia (No. III45005).

Conflicts of Interest: The authors declare no conflicts of interest.

\section{Abbreviations}

$\begin{array}{ll}\text { ALD } & \text { Alcoholic liver disease } \\ \text { ALT } & \text { Alanine aminotransferase } \\ \text { AST } & \text { Aspartate aminotransferase } \\ \text { BA } & \text { Bile acid } \\ \text { CHB } & \text { Chronic hepatitis B } \\ \text { CHC } & \text { Chronic hepatitis C } \\ \text { CLA } & \text { Conjugated linoleic acid } \\ \text { ESLD } & \text { End-stage liver disease } \\ \text { FMT } & \text { Farnesoid X receptor } \\ \text { FXR } & \text { Fecal microbiota transfer } \\ \text { GM } & \text { Gut microbiota } \\ \text { HCC } & \text { Hepatocellular carcinoma } \\ \text { HDL } & \text { High-density lipoprotein } \\ \text { HE } & \text { Hepatic encephalopathy } \\ \text { JNK } & \text { c-Jun N-terminal kinase } \\ \text { LGG } & \text { Lactobacillus GG AT strain 53103 } \\ \text { LPS } & \text { Lipopolysaccharides } \\ \text { MBOAT7 } & \text { Membrane bound O-acyltransferase domain } \\ \text { NAFLD } & \text { containing 7 } \\ \text { NASH } & \text { Non-alcoholic fatty liver disease } \\ \text { NFK } \beta & \text { Non-alcoholic steatohepatitis } \\ \text { NLRs } & \text { Nuclear factor kappa B } \\ \text { PAMPs } & \text { Nod-like receptors } \\ \text { PNPLA3 } & \text { Pathogen-associated molecular patterns } \\ \text { SLC38A4 } & \text { Patatin like phospholipase domain containing 3 } \\ \text { SBP } & \text { Solute carrier family 38 member 4 } \\ \text { SVR } & \text { Spontaneous bacterial peritonitis } \\ \text { TJ } & \text { Sustained virological response } \\ \text { TLRs } & \text { Tight junctions } \\ \text { TM6SF2 } & \text { Toll-like receptors } \\ & \text { Transmembrane 6 superfamily 2 human gene } \\ & \end{array}$

\section{References}

1. O'Hara, A.M.; Shanahan, F. The gut flora as a forgotten organ. EMBO J. 2006, 7, 688-693. [CrossRef] [PubMed]

2. Chassaing, B.; Etienne-Mesmin, L.; Gewirtz, A.T. Microbiota-liver axis in hepatic disease. Hepatology 2014, 59, 328-339. [CrossRef] [PubMed]

3. Tap, J.; Mondot, S.; Levenez, F.; Pelletier, E.; Caron, C.; Furet, J.P.; Ugarte, E.; Muñoz-Tamayo, R.; Paslier, D.L.; Nalin, R.; et al. Towards the human intestinal microbiota phylogenetic core. Environ. Microbiol. 2009, 11, 2574-2584. [CrossRef] [PubMed]

4. Kho, Z.Y.; Lal, S.K. The Human Gut Microbiome-A Potential Controller of Wellness and Disease. Front. Microbiol. 2018, 9, 1835. [CrossRef]

5. Tao, Z.; Siew, C.N. The Gut Microbiota in the Pathogenesis and Therapeutics of Inflammatory Bowel Disease. Front. Microbiol. 2018, 9, 2247. [CrossRef]

6. Russo, E.; Amedei, A. The Role of the Microbiota in the Genesis of Gastrointestinal Cancers. In Frontiers in Anti-Infective Drug Discovery; Bentham Science Publishers: Emirate of Sharjah, UAE, 2018; Volume 7. 
7. Al Khodor, S.; Shatat, F.I. Gut microbiome and kidney disease: A bidirectional relationship. Pediatr. Nephrol. 2017, 32, 921-931. [CrossRef] [PubMed]

8. Baothman, O.A.; Zamzami, M.A.; Taher, I.; Abubaker, J.; Abu-Farha, M. The role of Gut Microbiota in the development of obesity and Diabetes. Lipids Health Dis. 2016, 15, 108. [CrossRef]

9. Russo, E.; Bacci, G.; Chiellini, C.; Fagorzi, C.; Niccolai, E.; Taddei, A.; Ricci, F.; Ringressi, M.N.; Borrelli, R.; Melli, F.; et al. Preliminary Comparison of Oral and Intestinal Human Microbiota in Patients with Colorectal Cancer: A Pilot Study. Front. Microbiol. 2018, 8, 2699. [CrossRef]

10. Konturek, P.C.; Harsch, I.A.; Konturek, K.; Schink, M.; Konturek, T.; Neurath, M.F.; Zopf, Y. Gut-Liver Axis: How Do Gut Bacteria Influence the Liver? Med. Sci. (Basel) 2018, 6, 79. [CrossRef]

11. Vajro, P.; Paolella, G.; Fasano, A. Microbiota and gut-liver axis: A mini-review on their influences on obesity and obesity-related liver disease. J. Pediatr. Gastroenterol. Nutr. 2013, 56, 461-468. [CrossRef]

12. Yiu, J.H.; Dorweiler, B.; Woo, C.W. Interaction between gut microbiota and toll-like receptor: From immunity to metabolism. J. Mol. Med. 2017, 95, 13-20. [CrossRef]

13. Wang, J.; Wang, Y.; Zhang, X.; Liu, J.; Zhang, Q.; Zhao, Y.; Peng, J.; Feng, Q.; Dai, J.; Sun, S.; et al. Gut Microbial Dysbiosis Is Associated with Altered Hepatic Functions and Serum Metabolites in Chronic Hepatitis B Patients. Front. Microbiol. 2017, 8, 2222. [CrossRef]

14. Wei, X.; Yan, X.; Zou, D.; Yang, Z.; Wang, X.; Liu, W.; Wang, S.; Li, X.; Han, J.; Huang, L.; et al. Abnormal fecal microbiota community and functions in patients with hepatitis $B$ liver cirrhosis as revealed by a metagenomic approach. BMC Gastroenterol. 2013, 13, 175. [CrossRef] [PubMed]

15. Aly, A.M.; Adel, A.; El-Gendy, A.O.; Essam, T.M.; Aziz, R.K. Gut microbiome alterations in patients with stage 4 hepatitis C. Gut Pathog. 2016, 8, 42. [CrossRef]

16. Grat, M.; Wronka, K.M.; Krasnodebski, M.; Masior, L.; Lewandowski, Z.; Kosinska, I.; Grat, K.; Stypulkowski, J.; Rejowski, S.; Wasilewicz, M.; et al. Profile of gut microbiota associated with the presence of hepatocel- lular cancer in patients with liver cirrhosis. Transplant Proc. 2016, 48, 1687-1691. [CrossRef]

17. Zhang, H.L.; Yu, L.X.; Yang, W.; Tang, L.; Lin, Y.; Wu, H.; Zhai, B.; Tan, Y.X.; Shan, L.; Liu, Q.; et al. Profound impact of gut homeostasis on chemically induced pro-tumorigenic inflammation and hepatocarcinogenesis in rats. J. Hepatol. 2012, 57, 803-812. [CrossRef] [PubMed]

18. Häussinger, D.; Schliess, F. Pathogenetic mechanisms of hepatic encephalopathy. Gut 2008, 57, $1156-1165$. [CrossRef] [PubMed]

19. Dubinkina, V.B.; Tyakht, A.V.; Odintsova, V.Y.; Yarygin, K.S.; Kovarsky, B.A.; Pavlenko, A.V.; Ischenko, D.S.; Popenko, A.S.; Alexeev, D.G.; Taraskina, A.Y.; et al. Links of gut microbiota composition with alcohol dependence syndrome and alcoholic liver disease. Microbiome 2017, 5, 141. [CrossRef]

20. Chakraborti, C.K. New-found link between microbiota and obesity. World J. Gastrointest. Pathophysiol. 2015, 6, 110-119. [CrossRef]

21. Poeta, M.; Pierri, L.; Vajro, P. Gut-Liver Axis Derangement in Non-Alcoholic Fatty Liver Disease. Children 2017, 4, 66. [CrossRef]

22. Qin, N.; Yang, F.; Li, A.; Prifti, E.; Chen, Y.; Shao, L.; Guo, J.; Le Chatelier, E.; Yao, J.; Wu, L.; et al. Alterations of the human gut microbiome in liver cirrhosis. Nature 2014, 513, 59-64. [CrossRef] [PubMed]

23. Bajaj, J.S.; Heuman, D.M.; Hylemon, P.B.; Sanyal, A.J.; White, M.B.; Monteith, P.; Noble, N.A.; Unser, A.B.; Daita, K.; Fisher, A.R.; et al. Altered profile of human gut microbiome is associated with cirrhosis and its complications. J. Hepatol. 2014, 60, 940-947. [CrossRef] [PubMed]

24. Yang, R.; Xu, Y.; Dai, Z.; Lin, X.; Wang, H. The Immunologic Role of Gut Microbiota in Patients with Chronic HBV Infection. J. Immunol. Res. 2018, 2018, 2361963. [CrossRef] [PubMed]

25. Chou, H.H.; Chien, W.H.; Wu, L.L.; Cheng, C.H.; Chung, C.H.; Horng, J.H.; Ni, Y.H.; Tseng, H.T.; Wu, D.; $\mathrm{Lu}, \mathrm{X}$.; et al. Age-related immune clearance of hepatitis B virus infection requires the establishment of gut microbiota. Proc. Natl. Acad. Sci. USA 2015, 112, 2175-2180. [CrossRef] [PubMed]

26. Kang, Y.; Cai, Y. Gut microbiota and hepatitis-B-virus-induced chronic liver disease: Implications for faecal microbiota transplantation therapy. J. Hosp. Infect. 2017, 96, 342-348. [CrossRef]

27. Ren, Y.D.; Ye, Z.S.; Yang, L.Z.; Jin, L.X.; Wei, W.J.; Deng, Y.Y.; Chen, X.X.; Xiao, C.X.; Yu, X.F.; Xu, H.Z.; et al. Fecal microbiota transplantation induces hepatitis B virus e-antigen (HBeAg)clearance in patients with positive HBeAg after long-term antiviral therapy. Hepatology 2017, 65, 1765-1768. [CrossRef] [PubMed]

28. Chen, Y.; Yang, F.; Lu, H.; Wang, B.; Chen, Y.; Lei, D.; Wang, Y.; Zhu, B.; Li, L. Characterization of fecal microbial communities in patients with liver cirrhosis. Hepatology 2011, 54, 562-572. [CrossRef] 
29. Lee, M.H.; Yang, H.I.; Yuan, Y.; L'Italien, G.; Chen, C.J. Epidemiology and natural history of hepatitis C virus infection. World J. Gastroenterol. 2017, 20,9270-9280. [CrossRef]

30. Preveden, T.; Scarpellini, E.; Milić, N.; Luzza, F.; Abenavoli, L. Gut microbiota changes and chronic hepatitis C virus infection. Expert Rev. Gastroenterol. Hepatol. 2017, 11, 813-819. [CrossRef]

31. Heidrich, B.; Vital, M.; Plumeier, I.; Döscher, N.; Kahl, S.; Kirschner, J.; Ziegert, S.; Solbach, P.; Lenzen, H.; Potthoff, A.; et al. Intestinal microbiota in patients with chronic hepatitis $\mathrm{C}$ with and without cirrhosis compared with healthy controls. Liver Int. 2018, 38, 50-58. [CrossRef]

32. Bajaj, J.S.; Sterling, R.K.; Betrapally, N.S.; Nixon, D.E.; Fuchs, M.; Daita, K.; Heuman, D.M.; Sikaroodi, M.; Hylemon, P.B.; White, M.B.; et al. HCV eradication does not impact gut dysbiosis or systemic inflammation in cirrhotic patients. Aliment. Pharmacol. Ther. 2016, 44, 638-643. [CrossRef] [PubMed]

33. Munteanu, D.; Negru, A.; Radulescu, M.; Mihailescu, R.; Arama, S.S.; Arama, V. Evaluation of bacterial translocation in patients with chronic HCV infection. Rom. J. Intern. Med. 2014, 52, 91-96.

34. Cassard, A.M.; Ciocan, D. Microbiota, a key player in alcoholic liver disease. Clin. Mol. Hepatol. 2017, 24, 100-107. [CrossRef] [PubMed]

35. Hartmann, P.; Seebauer, C.T.; Schnabl, B. Alcoholic liver disease: The gut microbiome and liver cross talk. Alcohol Clin. Exp. Res. 2015, 39, 763-775. [CrossRef] [PubMed]

36. Szabo, G. Gut-liver axis in alcoholic liver disease. Gastroenterology 2014, 148, 30-36. [CrossRef] [PubMed]

37. Xie, G.; Zhong, W.; Zheng, X.; Li, Q.; Qiu, Y.; Li, H.; Chen, H.; Zhou, Z.; Jia, W. Chronic ethanol consumption alters mammalian gastrointestinal content metabolites. J. Proteome Res. 2013, 12, 3297-3306. [CrossRef] [PubMed]

38. Younossi, Z.M.; Marchesini, G.; Pinto-Cortez, H.; Petta, S. Epidemiology of Nonalcoholic Fatty Liver Disease and Nonalcoholic Steatohepatitis: Implications for Liver Transplantation. Transplantation 2018, 30335697. [CrossRef] [PubMed]

39. Boppidi, H.; Daram, S.R. Nonalcoholic fatty liver disease: Hepatic manifestation of obesity and the metabolic syndrome. Postgrad. Med. 2008, 120, 1-7. [CrossRef] [PubMed]

40. Gentric, G.; Maillet, V.; Paradis, V.; Couton, D.; L'Hermitte, A.; Panasyuk, G.; Fromenty, B.; Celton-Morizur, S.; Desdouets, C. Oxidative stress promotes pathologic polyploidization in nonalcoholic fatty liver disease. J. Clin. Investig. 2015, 125, 981-992. [CrossRef]

41. Zhu, L.; Baker, S.S.; Gill, C.; Liu, W.; Alkhouri, R.; Baker, R.D.; Gill, S.R. Characterization of gut microbiomes in nonalcoholic steatohepatitis (NASH) patients: A connection between endogenous alcohol and NASH. Hepatology 2013, 57, 601-609. [CrossRef]

42. Kakiyama, G.; Pandak, W.M.; Gillevet, P.M.; Hylemon, P.B.; Heuman, D.M.; Daita, K.; Takei, H.; Muto, A.; Nittono, H.; Ridlon, J.M.; et al. Modulation of the fecal bile acid profile by gut microbiota in cirrhosis. J. Hepatol. 2013, 58, 949-955. [CrossRef] [PubMed]

43. Sinal, C.J.; Tohkin, M.; Miyata, M.; Ward, J.M.; Lambert, G.; Gonzalez, F.J. Targeted disruption of the nuclear receptor FXR/BAR impairs bile acid and lipid homeostasis. Cell 2000, 102, 731-744. [CrossRef]

44. Goodwin, B.; Jones, S.A.; Price, R.R.; Watson, M.A.; McKee, D.D.; Moore, L.B.; Galardi, C.; Wilson, J.G.; Lewis, M.C.; Roth, M.E.; et al. A regulatory cascade of the nuclear receptors FXR, SHP-1, and LRH-1 represses bile acid biosynthesis. Mol. Cell. 2000, 6, 517-526. [CrossRef]

45. Leung, D.H.; Yimlamai, D. The intestinal microbiome and paediatric liver disease. Lancet Gastroenterol. Hepatol. 2017, 2, 446-455. [CrossRef]

46. Zhu, Y.; Li, F.; Guo, G.L. Tissue-specific function of farnesoid X receptor in liver and intestine. Pharmacol. Res. 2011, 63, 259-265. [CrossRef] [PubMed]

47. Shaik, F.B.; Prasad, D.V.; Narala, V.R. Role of farnesoid X receptor in inflammation and resolution. Inflamm. Res. 2015, 64, 9-20. [CrossRef] [PubMed]

48. Inagaki, T.; Moschetta, A.; Lee, Y.K.; Peng, L.; Zhao, G.; Downes, M.; Yu, R.T.; Shelton, J.M.; Richardson, J.A.; Repa, J.J.; et al. Regulation of antibacterial defense in the small intestine by the nuclear bile acid receptor. Proc. Natl. Acad. Sci. USA 2006, 103, 3920-3925. [CrossRef]

49. Oliveira, M.C.; Gilglioni, E.H.; Boer, B.A.; Waart, D.R.; Salgueiro, C.L.; Ishii-Iwamoto, E.L.; Oude Elferink, R.P.J.; Gaemers, I.C. Bile acid receptor agonists INT747 and INT777 decrease oestrogen deficiency-related postmenopausal obesity and hepatic steatosis in mice. Biochim. Biophys. Acta 2016, 1862, 2054-2062. [CrossRef] 
50. Chávez-Talavera, O.; Tailleux, A.; Lefebvre, P.; Staels, B. Bile acid control of metabolism and inflammation in obesity, type 2 diabetes, dyslipidemia, and nonalcoholic fatty liver disease. Gastroenterology 2017, 152, 1679-1694. [CrossRef]

51. Rai, R.; Saraswat, V.A.; Dhiman, R.K. Gut microbiota: Its role in hepatic encephalopathy. J. Clin. Exp. Hepatol. 2015, 5, 29-36. [CrossRef]

52. Oikonomou, T.; Papatheodoridis, G.V.; Samarkos, M.; Goulis, I.; Cholongitas, E. Clinical impact of microbiome in patients with decompensated cirrhosis. World J. Gastroenterol. 2018, 24, 3813-3820. [CrossRef] [PubMed]

53. Kulik, L.; El-Serag, H.B. Epidemiology and Management of Hepatocellular Carcinoma. Gastroenterology 2018. S0016-5085(18)35165-5. [CrossRef] [PubMed]

54. Nordenstedt, H.; White, D.L.; El-Serag, H.B. The changing pattern of epidemiology in hepatocellular carcinoma. Dig. Liver Dis. 2010, 42, 206-214. [CrossRef]

55. Cholankeril, G.; Patel, R.; Khurana, S.; Satapathy, S.K. Hepatocellular carcinoma in non-alcoholic steatohepatitis: Current knowledge and implications for management. World J. Hepatol. 2017, 9, 533-543. [CrossRef]

56. Wan, M.L.Y.; El-Nezami, H. Targeting gut microbiota in hepatocellular carcinoma: Probiotics as a novel therapy. Hepatobiliary Surg. Nutr. 2018, 7, 11-20. [CrossRef] [PubMed]

57. Tao, X.; Wang, N.; Qin, W. Gut Microbiota and Hepatocellular Carcinoma. Gastrointest. Tumors. 2015, 2, 33-40. [CrossRef]

58. Lu, H.; Ren, Z.; Li, A.; Zhang, H.; Jiang, J.; Xu, S.; Luo, Q.; Zhou, K.; Sun, X.; Zheng, S.; et al. Deep sequencing reveals microbiota dysbiosis of tongue coat in patients with liver carcinoma. Sci. Rep. 2016, 6, 33142. [CrossRef]

59. Dapito, D.H.; Mencin, A.; Gwak, G.Y.; Pradere, J.P.; Jang, M.K.; Mederacke, I.; Caviglia, J.M.; Khiabanian, H.; Adeyemi, A.; Bataller, R.; et al. Promotion of hepatocellular carcinoma by the intestinal microbiota and TLR4. Cancer Cell. 2012, 21, 504-516. [CrossRef]

60. Chalasani, N.; Younossi, Z.; Lavine, J.E.; Diehl, A.M.; Brunt, E.M.; Cusi, K.; Charlton, M.; Sanyal, A.J. The diagnosis and management of non-alcoholic fatty liver disease: Practice guideline by the American Gastroenterological Association, American Association for the Study of Liver Diseases, and American College of Gastroenterology. Gastroenterology 2012, 142, 1592-1609. [CrossRef]

61. Chang, B.; Sang, L.; Wang, Y.; Tong, J.; Zhang, D.; Wang, B. The protective effect of VSL\#3 on intestinal permeability in a rat model of alcoholic intestinal injury. BMC Gastroenterol. 2013, 13, 151. [CrossRef]

62. Ma, Y.Y.; Li, L.; Yu, C.H.; Shen, Z.; Chen, L.H.; Li, Y.M. Effects of probiotics on nonalcoholic fatty liver disease: A meta-analysis. World J. Gastroenterol. 2013, 19, 6911-6918. [CrossRef] [PubMed]

63. Bajaj, J.S.; Idilman, R.; Mabudian, L.; Hood, M.; Fagan, A.; Turan, D.; White, M.B.; Karakaya, F.; Wang, J.; Atalay, R.; et al. Diet Affects Gut Microbiota Modulates Hospitalization Risk Differentially In an International Cirrhosis Cohort. Hepatology. 2018, 68, 234-247. [CrossRef] [PubMed]

64. Kumar, M.; Verma, V.; Nagpal, R.; Kumar, A.; Gautam, S.K.; Behare, P.V.; Grover, C.R.; Aggarwal, P.K. Effect of probiotic fermented milk and chlorophyllin on gene expressions and genotoxicity during AFB1-induced hepatocellular carcinoma. Gene 2011, 490, 54-59. [CrossRef] [PubMed]

65. McGee, R.G.; Bakens, A.; Wiley, K.; Riordan, S.M.; Webster, A.C. Probiotics for patients with hepatic encephalopathy. Cochrane Database Syst. Rev. 2011, 11, CD008716. [CrossRef]

66. Holte, K.; Krag, A.; Gluud, L.L. Systematic review and meta-analysis of randomized trials on probiotics for hepatic encephalopathy. Hepatol. Res. 2012, 42, 1008-1015. [CrossRef] [PubMed]

67. Agrawal, A.; Sharma, B.C.; Sharma, P.; Sarin, S.K. Secondary prophylaxis of hepatic encephalopathy in cirrhosis: An open-label, randomized controlled trial of lactulose, probiotics, and no therapy. Am. J. Gastroenterol. 2012, 107, 1043-1050. [CrossRef]

68. Lunia, M.K.; Sharma, B.C.; Sharma, P.; Sachdeva, S.; Srivastava, S. Probiotics prevent hepatic encephalopathy in patients with cirrhosis: A randomized controlled trial. Clin. Gastroenterol. Hepatol. 2014, 12, 1003-1008. [CrossRef]

69. Shukla, S.; Shukla, A.; Mehboob, S.; Guha, S. Meta-analysis: The effects of gut flora modulation using prebiotics, probiotics and synbiotics on minimal hepatic encephalopathy. Aliment. Pharmacol. Ther. 2011, 33, 662-671. [CrossRef] [PubMed] 
70. Sajjad, A.; Mottershead, M.; Syn, W.K.; Jones, R.; Smith, S.; Nwokolo, C.U. Ciprofloxacin suppresses bacterial overgrowth, increases fasting insulin but does not correct low acylated ghrelin concentration in non-alcoholic steatohepatitis. Aliment. Pharmacol. Ther. 2005, 22, 291-299. [CrossRef]

71. Ferrere, G.; Wrzosek, L.; Cailleux, F.; Turpin, W.; Puchois, V.; Spatz, M.; Ciocan, D.; Rainteau, D.; Humbert, L.; Hugot, C.; et al. Fecal microbiota manipulation prevents dysbiosis and alcohol- induced liver injury in mice. J. Hepatol. 2017, 66, 806-815. [CrossRef]

72. Million, M.; Angelakis, E.; Paul, M.; Armougom, F.; Leibovici, L.; Raoult, D. Comparative meta-analysis of the effect of Lactobacillus species on weight gain in humans and animals. Microb Pathog. 2012, 53, 100-108. [CrossRef] [PubMed]

73. Gøbel, R.J.; Larsen, N.; Jakobsen, M.; Mølgaard, C.; Michaelsen, K.F. Probiotics to adolescents with obesity: Effects on inflammation and metabolic syndrome. J. Pediatr. Gastroenterol. Nutr. 2012, 55, 673-678. [CrossRef] [PubMed]

74. Larsen, N.; Vogensen, F.K.; Gøbel, R.J.; Michaelsen, K.F.; Forssten, S.D.; Lahtinen, S.J.; Jakobsen, M. Effect of Lactobacillus salivarius Ls-33 on fecal microbiota in obese adolescents. Clin. Nutr. 2013, 32, 935-940. [CrossRef] [PubMed]

75. Wang, W.W.; Zhang, Y.; Huang, X.B.; You, N.; Zheng, L.; Li, J. Fecal microbiota transplantation prevents hepatic encephalopathy in rats with carbon tetrachloride-induced acute hepatic dysfunction. World J. Gastroenterol. 2017, 23, 6983-6994. [CrossRef] [PubMed]

76. Adawi, D.; Kasravi, F.B.; Molin, G.; Jeppsson, B. Effect of Lactobacillus supplementation with and without arginine on liver damage and bacterial translocation in an acute liver injury model in the rat. Hepatology 1997, 25, 642-647. [CrossRef] [PubMed]

77. Philips, C.A.; Pande, A.; Shasthry, S.M.; Jamwal, K.M.; Khillan, V.; Chandel, S.S.; Kumar, G.; Sharma, M.K.; Maiwall, R.; Jindal, A.; et al. Healthy donor fecal microbiota transplantation in steroid-ineligible severe alcoholic hepatitis: A pilot study. Clin. Gastroenterol. Hepatol. 2017, 15, 600-602. [CrossRef] [PubMed]

78. Bajaj, J.S.; Kassam, Z.; Fagan, A.; Gavis, E.A.; Liu, E.; Cox, I.J.; Kheradman, R.; Heuman, D.; Wang, J.; Gurry, T.; et al. Fecal microbiota transplant from a rational stool donor improves hepatic encephalopathy: A randomized clinical trial. Hepatology 2017, 66, 1727-1738. [CrossRef]

79. Socha, P.; Horvath, A.; Vajro, P.; Dziechciarz, P.; Dhawan, A.; Szajewska, H. Pharmacological interventions for nonalcoholic fatty liver disease in adults and in children: A systematic review. J. Pediatr. Gastroenterol. Nutr. 2009, 48, 587-596. [CrossRef]

80. Minemura, M.; Shimizu, Y. Gut microbiota and liver diseases. World J. Gastroenterol. 2015, 21, $1691-1702$. [CrossRef]

81. Li, Z.; Yang, S.; Lin, H. Probiotics and antibodies to TNF inhibit inflammatory activity and improve nonalcoholic fatty liver disease. Hepatology 2003, 37, 343-350. [CrossRef]

82. Chen, L.; Pan, D.D.; Zhou, J. Protective effect of selenium-enriched Lactobacillus on CCl4-induced liver injury in mice and its possible mechanisms. World J. Gastroenterol. 2005, 11, 795-800. [CrossRef]

83. Velayudham, A.; Dolganiuc, A.; Ellis, M. VSL\#3 probiotic treatment attenuates fibrosis without changes in steatohepatitis in a diet-induced non-alcoholic steatohepatitis model in mice. Hepatology 2009, 49, 989-997. [CrossRef] [PubMed]

84. Ma, X.; Hua, J.; Li, Z. Probiotics improve high fat diet-induced hepatic steatosis and insulin resistance by increasing hepatic NKT cells. J. Hepatol. 2008, 49, 821-830. [CrossRef] [PubMed]

85. Aller, R.; De Luis, D.A.; Izaola, O.; Conde, R.; Gonzalez, S.M.; Primo, D.; De La Fuente, B.; Gonzalez, J. Effect of a probiotic on liver aminotransferases in nonalcoholic fatty liver disease patients: A double blind randomized clinical trial. Eur. Rev. Med. Pharmacol. Sci. 2011, 15, 1090-1095. [PubMed]

86. Wall, R.; Marques, T.M.; O'Sullivan, O.; Ross, R.P.; Shanahan, F.; Quigley, E.M.; Dinan, T.G.; Kiely, B.; Fitzgerald, G.F.; Cotter, P.D.; et al. Contrasting effects of Bifidobacterium breve NCIMB 702258 and Bifidobacterium breve DPC 6330 on the composition of murine brain fatty acids and gut microbiota. Am. J. Clin. Nutr. 2012, 95, 1278-1287. [CrossRef] [PubMed]

87. Malaguarnera, M.; Vacante, M.; Antic, T.; Giordano, M.; Chisari, G.; Acquaviva, R.; Mastrojeni, S.; Malaguarnera, G.; Mistretta, A.; Li Volti, G.; et al. Bifidobacterium longum with fructo-oligosaccharides in patients with non alcoholic steatohepatitis. Dig. Dis. Sci. 2012, 57, 545-553. [CrossRef] 
88. Tannock, G.W.; Wilson, C.M.; Loach, D.; Cook, G.M.; Eason, J.; O’Toole, P.W.; Holtrop, G.; Lawley, B. Resource partitioning in relation to cohabitation of Lactobacillus species in the mouse forestomach. ISME J. 2012, 6, 927-938. [CrossRef]

89. Cani, P.D.; Possemiers, S.; Van de Wiele, T.; Guiot, Y.; Everard, A.; Rottier, O.; Geurts, L.; Naslain, D.; Neyrinck, A.; Lambert, D.M.; et al. Changes in gut microbiota control inflammation in obese mice through a mechanism involving GLP-2-driven improvement of gut permeability. Gut 2009, 58, 1091-1103. [CrossRef]

90. Gunnarsdottir, S.A.; Sadik, R.; Shev, S.; Simrén, M.; Sjövall, H.; Stotzer, P.O.; Abrahamsson, H.; Olsson, R.; Björnsson, E.S. Small intestinal motility disturbances and bacterial overgrowth in patients with liver cirrhosis and portal hypertension. Am. J. Gastroenterol. 2003, 98, 1362-1370. [CrossRef]

91. Tarantino, G.; Finelli, C. Systematic review on intervention with prebiotics/probiotics in patients with obesity-related nonalcoholic fatty liver disease. Future Microbiol. 2015, 10, 889-902. [CrossRef]

92. Musa-Veloso, K.; Venditti, C.; Lee, H.Y.; Darch, M.; Floyd, S.; West, S.; Simon, R. Systematic review and meta-analysis of controlled intervention studies on the effectiveness of long-chain $\Omega-3$ fatty acids in patients with nonalcoholic fatty liver disease. Nutr. Rev. 2018, 76, 581-602. [CrossRef] [PubMed]

93. Robertson, R.C.; Kaliannan, K.; Strain, R.; Ross, R.P.; Stanton, C.; Kang, J.X. Maternal $\Omega-3$ fatty acids regulate offspring obesity through persistent modulation of gut microbiota. Microbiome 2018, 6, 95. [CrossRef] [PubMed]

94. Costantini, L.; Molinari, R.; Farinon, B.; Merendino, N. Impact of omega-3 fatty acids on the gut microbiota. Int. J. Mol. Sci. 2017, 18, 2645. [CrossRef] [PubMed]

95. Bajaj, J.S.; Fagan, A.; Sikaroodi, M.; White, M.B.; Sterling, R.K.; Gilles, H.; Heuman, D.; Stravitz, R.T.; Matherly, S.C.; Siddiqui, M.S.; et al. Liver transplant modulates gut microbial dysbiosis and cognitive function in cirrhosis. Liver Transpl. 2017, 23, 907-914. [CrossRef] [PubMed]

96. Stadlbauer, V.; Mookerjee, R.P.; Hodges, S.; Wright, G.A.K.; Davies, N.A.; Jalan, R. Effect of probiotic treatment on deranged neutrophil function and cytokine responses in patients with compensated alcoholic cirrhosis. J. Hepatol. 2008, 48, 945-951. [CrossRef] [PubMed]

97. LoGuercio, C.; Federico, A.; Tuccillo, C.; Terracciano, F.; D’Auria, M.V.; De Simone, C.; Del Vecchio Blanco, C. Beneficial effects of a probiotic VSL\#3 on parameters of liver dysfunction in chronic liver diseases. J. Clin. Gastroenterol. 2005, 39, 540-543. [PubMed]

98. Bajaj, J.S.; Heuman, D.M.; Hylemon, P.B.; Sanyal, A.J.; Puri, P.; Sterling, R.K.; Luketic, V.; Stravitz, R.T.; Siddiqui, M.S.; Fuchs, M.; et al. Randomised clinical trial: Lactobacillus GG modulates gut microbiome, metabolome and endotoxemia in patients with cirrhosis. Aliment. Pharmacol. Ther. 2014, 39, 1113-1125. [CrossRef]

99. Yoshimoto, S.; Loo, T.M.; Atarashi, K.; Kanda, H.; Sato, S.; Oyadomari, S.; Iwakura, Y.; Oshima, K.; Morita, H.; Hattori, M.; et al. Obesity-induced gut microbial metabolite promotes liver cancer through senescence secretome. Nature 2013, 52, 97-101. [CrossRef]

100. Huang, L.; Duan, C.; Zhao, Y.; Gao, L.; Niu, C.; Xu, J.; Li, S. Reduction of Aflatoxin B1 Toxicity by Lactobacillus plantarum C88: A Potential Probiotic Strain Isolated from Chinese Traditional Fermented Food “Tofu". PLoS ONE 2017, 12, e0170109. [CrossRef]

(C) 2019 by the authors. Licensee MDPI, Basel, Switzerland. This article is an open access article distributed under the terms and conditions of the Creative Commons Attribution (CC BY) license (http://creativecommons.org/licenses/by/4.0/). 